\title{
ANÁlISE DA PRODUÇÃo CIENTÍFICA DA REVISTA DE ADMINISTRAÇÃO E INOVAÇÃO
}

\author{
Henrique César Melo Ribeiro \\ Doutorando em Administração de Empresas pela Universidade Nove de Julho - UNINOVE \\ Professor da Faculdade Piauiense - FAP \\ hcmribeiro@hotmail.com (Brasil)
}

\section{Claudia Brito Silva Cirani}

Doutora em Economia Aplicada pela Escola Superior de Agricultura Luiz de Queiroz / Universidade de São Paulo - ESALQ / USP

Professora da Universidade Nove de Julho - UNINOVE

cloubrito@hotmail.com (Brasil)

\section{Elizabeth Jorge da Silva Monteiro de Freitas}

Doutora em Administração na Universidade Federal da Bahia - UFBA

elizabethmfreitas@gmail.com (Brasil)

\section{RESUMO}

Este artigo investigou a produção científica brasileira da Revista de Administração e Inovação, por meio de pesquisa bibliométrica e sociométrica, utilizando-se de estatística descritiva realizada no acervo da revista. Foram analisados 216 artigos, de 2006 a 2012. As principais conclusões deste trabalho foram: Pereira, R da S, Pereira, M. F., e Gonçalves, C. A. possuem alta centralidade de grau. Em relação à centralidade de intermediação, destacam-se os autores Nohara, J. J., Pereira, M. F., e Esteves, G. As Instituições de Ensino SuperiorUSP, Uninove, FGV (SP) e UPM possuem alta centralidade de grau. De maneira geral, houve baixa centralização da rede social de instituições e da rede de coautoria. As obras de Porter, Yin, Hamel, Schumpeter e Nelson foram as mais citadas nas publicações. Os temas que mais apareceram durante os sete anos analisados foram: estudos setoriais e tecnologia; organização e de negócios; redes organizacionais; gestão do conhecimento; e empreendedorismo e PMEs.

Palavras-chave: Inovação; Administração; Bibliometria; Sociometria. 


\section{INTRODUÇÃO}

O desenvolvimento, a disseminação e a utilização do conhecimento em qualquer área acadêmica dependem de forma significativa da circulação de ideias por meio das publicações de livros didáticos e, principalmente, de revistas científicas (Hoffman \& Holbrook, 1993). Nesse contexto, ressalta-se a relevância da Revista de Administração e Inovação (RAI), cuja missão é divulgar a produção intelectual na área de inovação tecnológica, organizacional e de mercado, estimulando as contribuições criativas e inéditas do trabalho acadêmico e de pesquisa. Destacam-se como objetivos da revista:

Contribuir para o aumento da produção de conhecimento das comunidades acadêmicas e profissional nas Ciências Sociais Aplicadas na área de inovação; servir como canal adequado para difundir avanços conceituais, metodológicos e de experiências de inovação na sociedade moderna; e estimar a difusão de conhecimentos que promovem novos estudos e novas formulações teóricas e empíricas para a área de inovação (Revista de Administração e Inovação [RAI], 2012).

Em 2004 a RAI iniciou as suas publicações e, entre 2004 a 2012, 244 artigos foram publicados em nove volumes. O objetivo deste trabalho é fornecer um mapeamento sobre o que tem sido pesquisado e publicado na RAI, por meio das análises bibliométrica e sociométrica, cuja técnica de pesquisa não só analisa publicações em periódicos científicos (Ferreira, 2011), como também analisa as redes sociais (Ramos-Rodríguez \& Ruíz-Navarro, 2004) a fim de compreender as ligações e conectividades entre autores e suas respectivas Instituições de Ensino Superior (IES).

Assim, na utilização da bibliometria, uma técnica que mensura o fluxo de informação (Francisco, 2011), apoiada pelos métodos quantitativos (Borges, 2002), matemáticos (Alvarenga, 1998) e estatísticos (Ortiz, 2002), utilizam-se como metodologias o agrupamento biblioFigura e a análise das palavras-chave (Francisco, 2011). Estudos de coautoria são também utilizados visando a entender a produção científica por meio da análise da parceria entre os autores (Parreiras, Silva, Matheus \& Brandão, 2006) e da interação de suas respectivas IES, evidenciando a produção científica intelectual no meio acadêmico.

A análise bibliométrica e de redes sociais no meio acadêmico desempenha papel fundamental para o fomento, a disseminação e a socialização do conhecimento científico. É fato que o número de estudos bibiliométricos e sociométricos na exploração de acervos de periódicos nacionais e internacionais tem aumentado significativamente em várias áreas do conhecimento.

Ramos-Rodríguez and Ruíz-Navarro (2004), por exemplo, identificaram as obras que tiveram o maior impacto sobre a estratégia em publicações de Administração no Strategic Management Journal, 
de 1980 a 2000. O estudo constatou que Michael Porter foi o autor mais evidenciado e que grupos de pesquisadores partilham os mesmos interesses, influenciando nas citações das mesmas referências.

McMillan and Casey (2007) realizaram uma análise bibliométrica do Journal British Journal of Industrial Relations entre os anos de 1986 a 2005. Observaram uma maturidade das publicações em 20 anos de pesquisa, constatando que, de 1986 a 1990, os trabalhos basearam-se na literatura econômica e, a posteriori, na de recursos humanos.

Ullah, Butt and Haroon (2008) avaliaram bibliometricamente os artigos do Journal of Ayub Medical College de 1997 a 2006, concluindo que o número de artigos publicados por ano variou de 27 a 97, com uma maioria de 11 a 20 citações, em que se visualizam os autores mais prolíferos, cujas contribuições foram de sete publicações.

Ramos-Rodríguez and Ruíz-Navarro (2004) e Fernandez-Alles and Ramos-Rodríguez (2009), de modo similar, analisaram a estrutura intelectual do tema gestão de recursos humanos por meio da técnica da bibliometria no periódico Journal Human Resource Management de 1985 a 2005. Observaram que as citações de livros são superiores às de revistas, constatando que as publicações com 2 ou mais pesquisadores prevalecem.

Ordóñez, Hernández, Hernández e Méndez (2009) analisaram na revista de Economía Institucional em seus primeiros 10 anos, constatando que o autor mais profícuo publicou 5 artigos, identificando uma certa homogenia entre as IES.

Durisin, Calabretta and Parmeggiani (2010) investigaram o Journal of Product Innovation Management por meio da bibliometria entre os anos de 1984 e 2004. Observaram eles um forte impacto das obras mais influentes e maturidade das pesquisas sobre inovação no período analisado.

Pinto, Santos e Santos (2009) analisaram as citações da revista eletrônica Arquivística.Net no período de 2005 a 2007, evidenciando os seguintes resultados: predominância de autoria sem parceria; livros como sendo as fontes mais citadas nas referências; e período médio de cobertura da literatura citada de 4 a 5 anos.

Explorando a área de empreendedorismo, Borba, Hoeltgebaum e Silveira (2011) analisaram a produção científica do periódico Academy of Management Meeting de 1954 a 2005,verificando que poucos autores publicam muito e são citados muitas vezes.

Francisco (2011) explorando o acervo da revista RAE-eletrônica de 2002 a 2010, por meio da bibliometria, análise de redes sociais e análise geográfica, concluiu que a média de autores por artigo foi superior a dois. $\mathrm{O}$ autor mais profícuo publicou cinco artigos, e a grande maioria das pesquisas foi escrita por autores pertencentes a uma única IES.

Revista de Administração e Inovação, São Paulo, v. 10, n.4, p. 208-228, out./dez. 2013. 
Leal, Aguillera e Melo-Silva (2011) realizaram estudo semelhante em 137 artigos publicados na Revista Psicologia: Organizações e Trabalho. Os principais resultados mostraram um predomínio de pesquisa de autoria múltipla, com uma grande parcela de autores do gênero feminino e de artigos voltados à prática e ao desenvolvimento de estratégias.

Portanto, são cada vez mais comuns estudos utilizando técnicas bibliométricas para analisar periódicos (Francisco, 2011). A RAI abordou o tema recentemente, por meio do uso de técnicas de bibliometria em alguns de seus artigos publicados: Bobsin, Visentini e Rech (2009), Nassif, Silva, Ono, Bontempo e Tinoco (2010), Peleias, Wahlmann, Parisi e Antunes (2010), Andrighi, Hoffmann e Andrade (2011), Farber, Hoeltgebaum e klemz (2011), Gomes, Machado Neto e Giotto (2011) e Vitorino Filho, Sacomano Neto, Silva e Guiliani (2012).

Porém, ainda não existem estudos sobre o que tem sido pesquisado e publicado na própria RAI. Dessa forma, este trabalho teve como objetivo fornecer um mapeamento sobre autoria, redes de coautoria, referências mais citadas, rede das IES, abordagens metodológicas, frequência de palavraschave, temas abordados, entre outras características, que predominam nas pesquisas relatadas nos artigos levantados na RAI.

Em síntese, o artigo em questão está organizado em quatro partes. A fundamentação teórica que sustenta o estudo constitui a parte dois, seguida pela três, em que se descrevem os procedimentos metodológicos usados na pesquisa e, em seguida, são apresentadas a análise e discussão dos resultados. Por fim, as considerações finais, bem como as limitações da pesquisa e as recomendações para estudos futuros.

\section{FUNDAMENTAÇÃO TEÓRICA}

As análises para fornecer um mapeamento sobre o que tem sido pesquisado e publicado sobre o tema inovação na RAI foram feitas com base nas três leis bibliométricas mais utilizadas em trabalhos desse escopo, tais como: a Lei de Bradford que enfatiza a produtividade dos periódicos. Ressalta-se, portanto, o grau de relevância da revista como um aspecto bastante importante; a Lei de Lotka, que destaca a produtividade científica de autores, ou seja, o número de vezes que cada um aparece citado em trabalhos científicos, por meio de um modelo de distribuição de tamanho-frequência em um conjunto de pesquisas, evidenciando aspectos de coautoria; e a Lei de Zipf, que conta as incidências de palavras nos artigos científicos, como por exemplo, as palavras-chave mais visualizadas nos artigos investigados. Este estudo foi conduzido com base nessas três leis.

Revista de Administração e Inovação, São Paulo, v. 10, n.4, p. 208-228, out./dez. 2013. 
Complementando a investigação bibliométrica, também foi utilizado neste trabalho a sociometria, isto é, o monitoramento das redes de coautoria (Ramos-Rodríguez \& Ruíz-Navarro, 2004) a fim de compreender as ligações e conectividades entre autores e suas respectivas IES.

O conceito de redes pode auxiliar na compreensão dos processos de interação social entre os atores e da própria geração do conhecimento. Nesse sentido, redes são um conjunto de nós e suas relações proporcionando interações e organizações sociais, em que a informação e o conhecimento são elementos-chave desse processo (Didriksson, 2003).

Também pode-se entender a rede social como um conjunto de pessoas, instituições ou organizações que, por possuírem afinidades em comum, compartilham, por exemplo, trabalho e/ou informações e, por meio dessas ligações, constroem e reconstroem uma estrutura social (Tomaél \& Marteleto, 2006).

Sebastián (1999, p. 309) aprofunda esse conceito, trazendo-o para o campo da pesquisa científica como "... modalidade de redes de cooperação e [que] se definem pela associação de grupos de pesquisa para a realização de trabalhos conjuntos, geralmente através de projetos de Pesquisa e Desenvolvimento".

Para se analisar uma rede social, é necessária a compreensão da sua estrutura, assim como das relações que a compõem. A principal propriedade estrutural da rede é a densidade que, segundo Gnyawali and Madhavan (2001), pode ser entendida a partir da extensão da interconexão entre os atores, ou seja, quanto maior a interconexão, maior a densidade. Tratando-se das relações, a coesão entre os atores é o indicador mais representativo. As medidas de centralidade, densidade e transitividade (probabilidade de duas pessoas estarem conectadas), compõem algumas das principais propriedades estruturais de uma rede.

A centralidade de grau (Degree) é definida pelo número de laços adjacentes de um ator com relação aos outros numa rede (Wasserman \& Faust, 1994), possibilitando uma avaliação da atividade local dos atores. A centralidade de proximidade (Closeness) é função da proximidade ou distância de um ator em relação a todos os outros numa rede.

A ideia percebida na análise desse indicador é que um autor com elevada centralidade de aproximação é aquele que possui maiores condições de interagir rapidamente com todos os outros (Hanneman \& Riddle, 2005; Scott, 2000; Wasserman \& Faust, 1994).

Por fim, a centralidade de intermediação (Betweenness) avalia a dependência de atores não adjacentes de outros que atuam como uma espécie de ponte para efetivação da interação deles (Freeman, 1992). Nesse caso, quanto maior o grau de centralidade de intermediação, maior a probabilidade de controle de um ator sobre outros que dele dependem para executar a interação. Redes Revista de Administração e Inovação, São Paulo, v. 10, n.4, p. 208-228, out./dez. 2013. 
de conhecimento, assim como a maioria das redes sociais, têm na coesão um dos seus principais elementos de análise. Assim, no comportamento coletivo dos atores de um mesmo grupo, a coesão serve de base para solidariedade e identidade do grupo.

Estruturalmente, as redes podem ser densas ou difusas e, relacionalmente, podem ter conexões fortes ou fracas. Conexões fracas e redes difusas são mais típicas nos ambientes instáveis, pois permitem maior fluxo de novas informações; enquanto que conexões fortes e redes densas são comuns aos ambientes estáveis, já que a troca de informações é refinada, com um alto nível de confiança, cooperação, reciprocidade e controle social entre os atores (Rowley, Behrens \& Krackhardt, 2000), podendo, entretanto, conduzir ao isolamento do grupo e a uma possível dificuldade de acesso desses atores a novas informações que circulem fora daquele ambiente restrito.

\section{PROCEDIMENTOS METODOLÓGICOS}

O procedimento metodológico utilizado neste trabalho consiste em uma análise bibliométrica e sociométrica (Vanti, 2002), de natureza qualitativa e descritiva (Francisco, 2011), cujo objetivo é o de identificar as seguintes características predominantes nos artigos analisados: autoria, redes de coautoria, obras mais citadas, redes de IES, tipo de abordagem e de técnica de pesquisa, palavraschave mais utilizadas nos artigos, classificação dos artigos por temas, e autores e IES que mais publicaram artigos.

Para mapear as pesquisas científicas da RAI, foram levantados dados e informações bibliográficas por meio do uso dos softwares UCINET 6 for Windows (versão 6.357) e Microsoft Excel 2007 a fim de construir as figuras e representações gráficas.

A população alvo deste trabalho abrange todos os artigos da RAI, publicados no período de 2006 a 2012, englobando o acervo mais recente disponibilizado pela revista, na época em que se realizou este trabalho. Ressalta-se que não foram considerados os dois primeiros anos - 2004 e 2005 já que o periódico estava em sua fase inicial, isto é, a RAI estava passando pelas etapas de padronização, classificação e indicação pela área de avaliação para participar no Qualis da Coordenação de Aperfeiçoamento de Pessoal de Nível Superior (CAPES). Portanto, achou-se conveniente para este estudo, não utilizar os 28 primeiros artigos publicados na RAI, mitigando assim uma possível endogenia de IES, docentes ou pesquisadores que publicaram artigos científicos nos primeiros dois anos do periódico. O site da revista - http://www.revistarai.org/- foi utilizado como fonte de coleta dos dados

Revista de Administração e Inovação, São Paulo, v. 10, n.4, p. 208-228, out./dez. 2013. 


\section{ANÁLISE E DISCUSSÃO DOS RESULTADOS}

A partir do levantamento de dados, foi possível identificar 216 artigos, numa constatação visível do aumento das publicações nos últimos três anos, conforme apresentado na Figura 1. O aumento de $88 \%$ em 2012 em relação ao período de 2007-2009 pode ser resultado do aumento do interesse pelo tema da inovação no meio acadêmico.

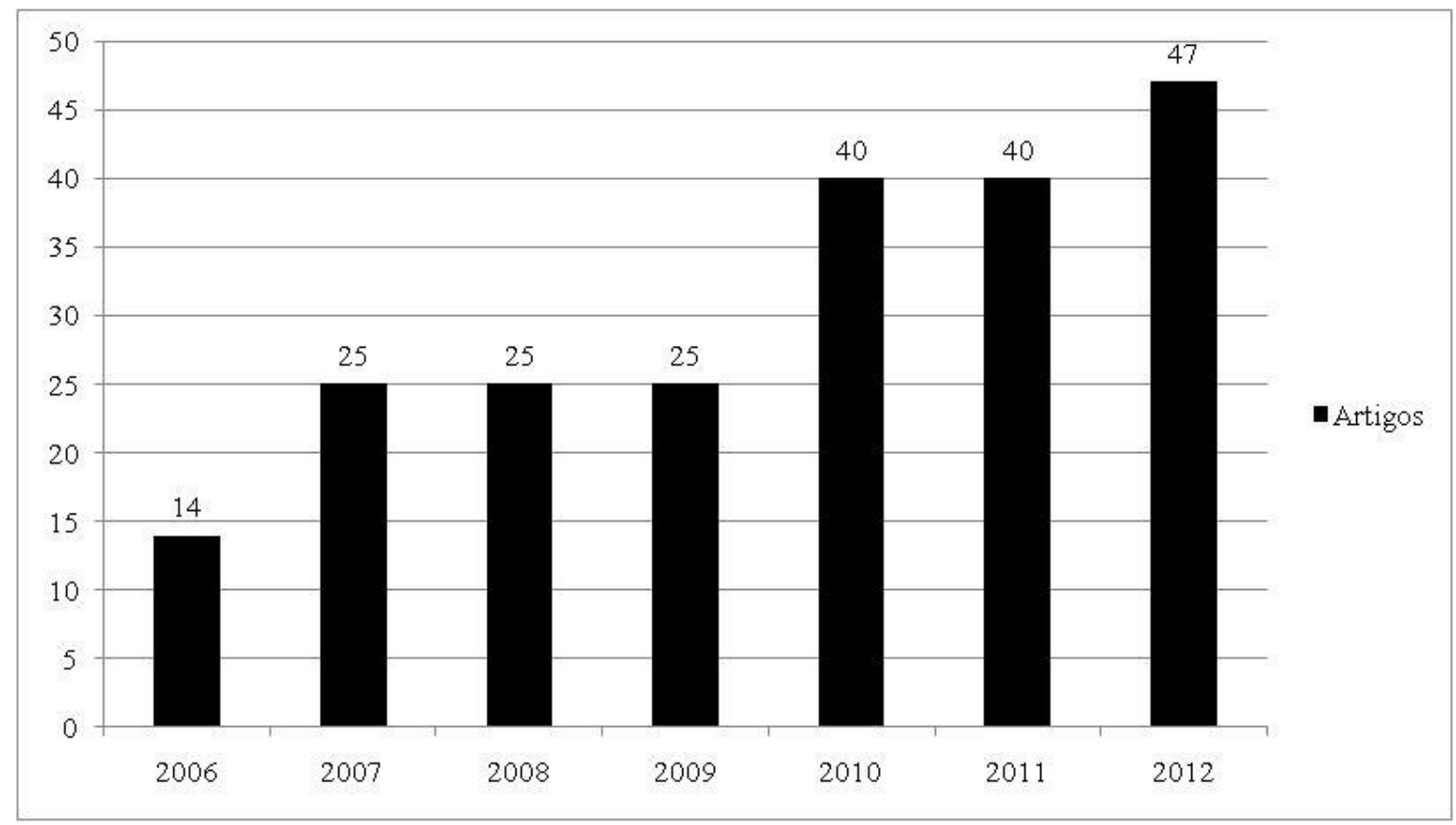

Figura 1. Artigos publicados na RAI por ano de publicação - 2006/2012 Fonte: Dados da pesquisa

Em relação ao número de autores por artigo, como se pode observar na Figura 2, prevalece o número de artigos publicados com dois a cinco autores, representando aproximadamente $91 \%$ do universo. Ressalta-se que a média de autoria ao longo dos sete anos de pesquisa foi de 2,71 autores por artigo publicado, como se pode visualizar na Figura 3. 


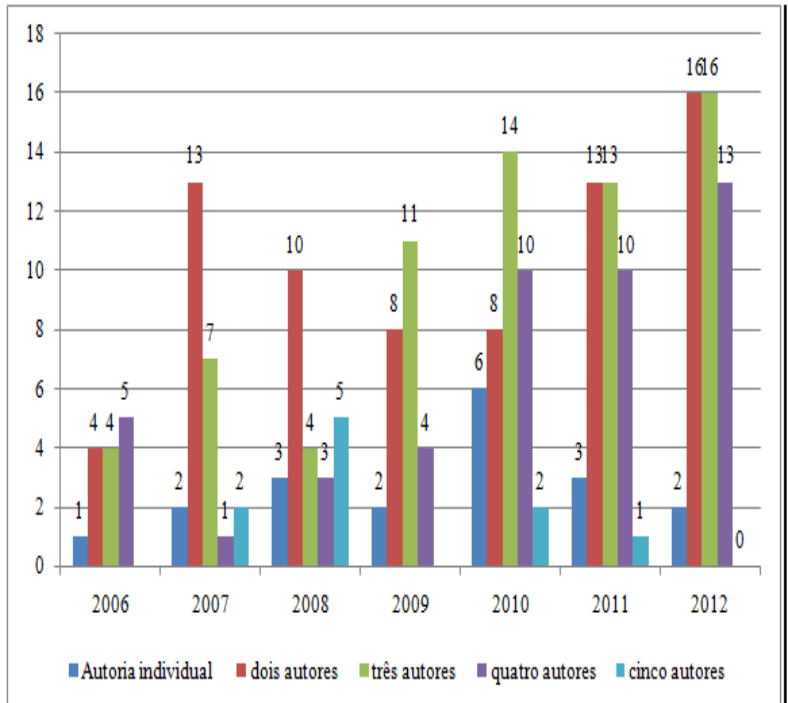

Figura 2. Características de autoria Fonte: Dados da pesquisa

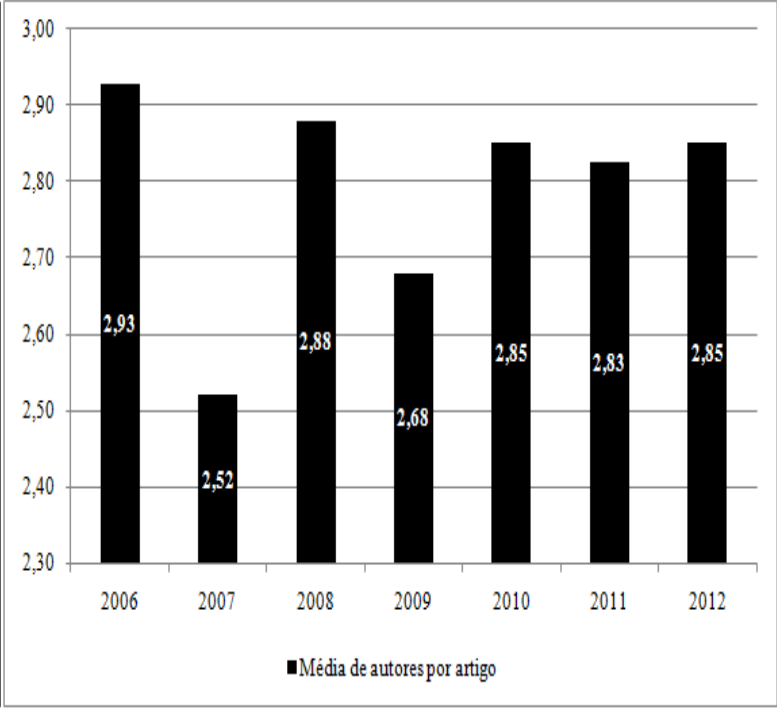

Figura 3. Média de autores por artigo Fonte: Dados da pesquisa

Esses resultados podem sugerir o início da consolidação de pequenos grupos de pesquisa, contribuindo assim para o crescimento da conectividade entre os autores e suas respectivas IES.

A Figura 4 mostra as redes de coautores dos artigos analisados na RAI no período de 2006 a 2012.

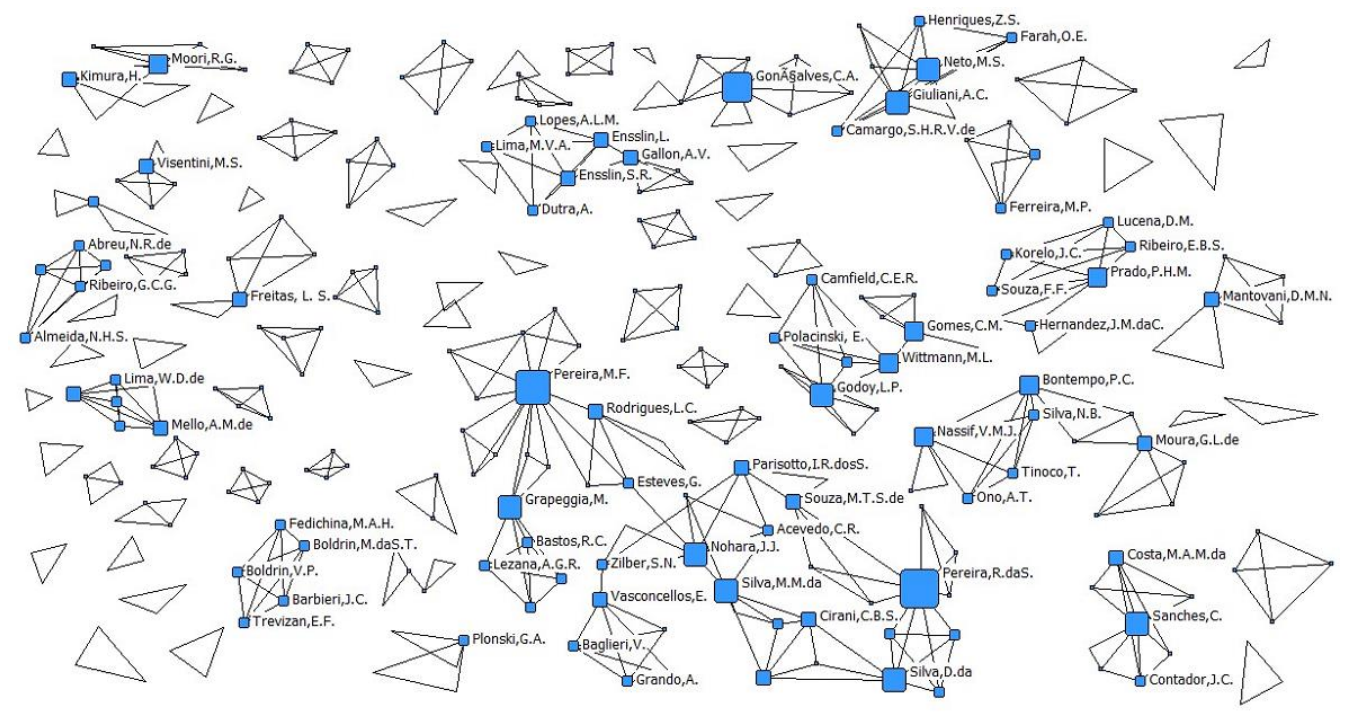

Figura 4. Redes de coautoria na RAI - 2006/2012

Fonte: Dados da pesquisa

Verifica-se pela Figura 4 que a rede social dos autores configura-se com baixa interação, isto é, revela baixa centralização. Esse resultado pode ser corroborado quando se constata que a densidade total do grupo é de 0,0056 , ou seja, a rede realiza $0,56 \%$ do total de suas relações. Tal cenário não Revista de Administração e Inovação, São Paulo, v. 10, n.4, p. 208-228, out./dez. 2013. 
reflete o ideal quando se trata de redes de coautoria, já que os grupos encontram-se isolados, inexistindo atores para fazer a ponte, ou seja, uma conexão entre eles. Esses atores seriam responsáveis pela troca de conhecimento não redundante entre os grupos. Os estudos de Ordóñez et al. (2009) e de Francisco (2011) sobre redes de coautoria atestam as informações visualizadas na Figura 1, pois, observaram em seus respectivos estudos pouca interação entre os atores envolvidos nos trabalhos.

Ao se analisar os autores que se destacam na rede, percebe-se a importância de alguns como: Pereira, R. da S., Pereira, M. F., e Gonçalves, C. A. Esses autores possuem alta centralidade de grau (degree), o que indica o maior número de relações na rede, ou seja, são aqueles que são mais procurados ou que procuram mais parceiros para a produção e publicação de artigos na RAI.

$\mathrm{Na}$ análise da centralidade de intermediação (betweenness) na Figura 5, percebe-se a dispersão da rede, pois poucos atores representam essa medida, tendo a maior parte localizada no seu componente principal. Deve-se destacar que os autores Nohara, J. J., Pereira, M. F., Esteves, G., Parisotto, I. R. dos S., e Souza, M. T. S. de, são os maiores responsáveis pela troca da informação na rede.

Ressalta-se, especialmente, a importância da participação do autor Pereira, M. F. visto que possui alta centralidade de grau, assim como alta centralidade de intermediação.

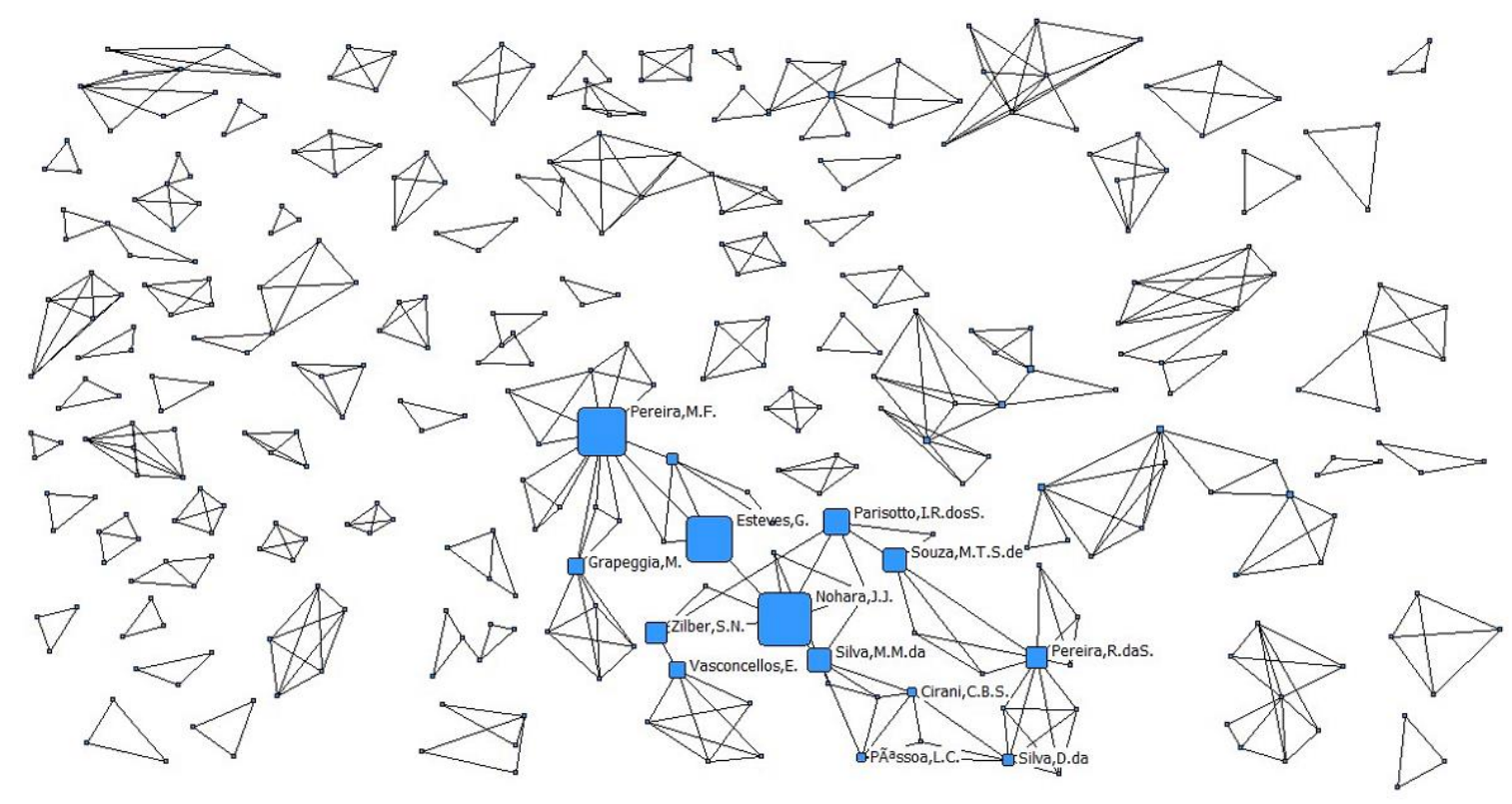

Figura 5. Centralidade de intermediação na RAI - 2006/2012 Fonte: Dados da pesquisa

A análise das citações é um indicador útil para verificar as obras que têm maior influência sobre a pesquisa existente.

Revista de Administração e Inovação, São Paulo, v. 10, n.4, p. 208-228, out./dez. 2013. 


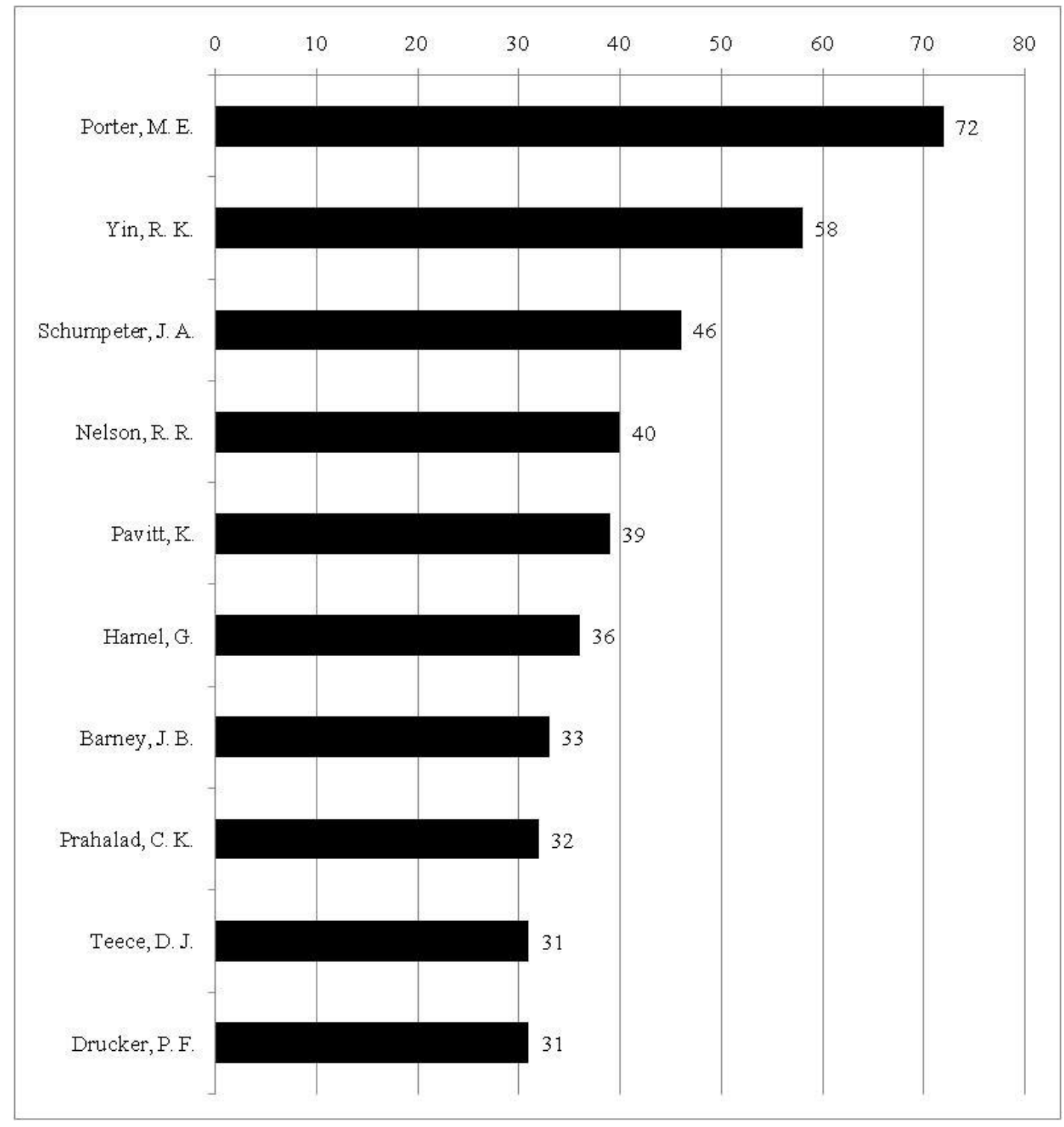

Figura 6. Autores mais citados na RAI - 2006/2012

Fonte: Dados da pesquisa

A Figura 6 mostra a frequência dos pesquisadores mais citados no acervo de 2006 a 2012 da RAI. Conforme a Figura 4, Porter, M. E. é o autor mais citado nas referências, com 72 citações. Em seguida, Yin, R. K., com 58 citações, o que demonstra que um grande número de artigos publicados utilizam a metodologia de análise de casos.

Podem-se verificar, também, outros autores mais citados nas referências dos 216 artigos pesquisados, tais como: Schumpeter, J. A. (com 46 citações), Nelson, R. R. (40 citações), Pavitt, K. (39), Hamel, G. (36), Barney, J. B. (33), Prahalad, C. K. (32), e Teece, D. J. e Drucker, P. F., ambos 
citados 31 vezes. Esses resultados apoiam uma das leis bibliométricas utilizadas neste trabalho: a Lei de Lotka.

A Figura 7 mostra as principais redes das IES dos 216 artigos publicados ao longo dos sete anos da Revista de Administração e Inovação.

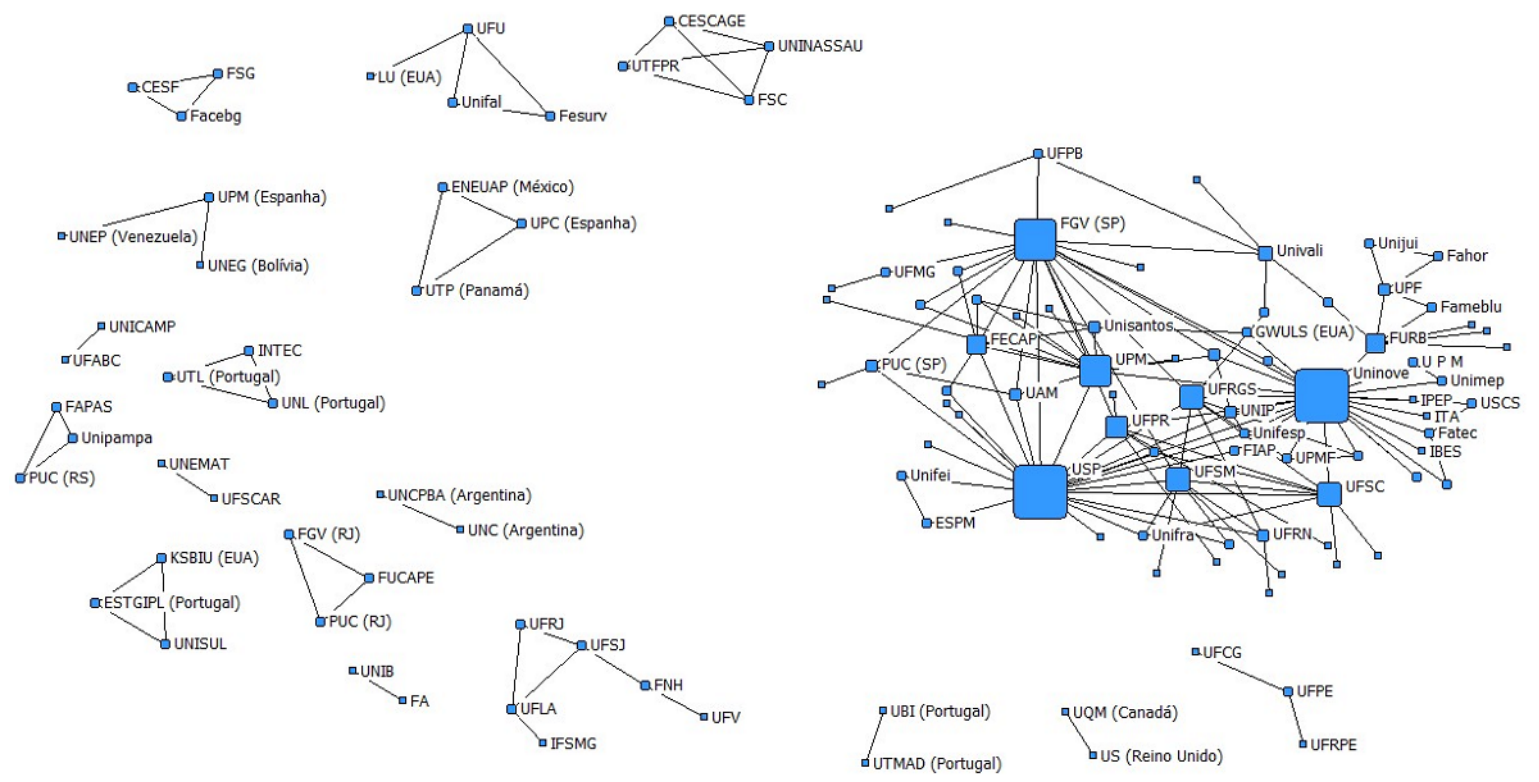

Figura 7. Rede das IES da RAI - 2006/2012

Fonte: Dados da pesquisa

É possível observar pela Figura 7 que a rede de IES apresentou baixa centralização representada por sua baixa densidade, equivalente a 0,0232 , ou seja, apenas $2,32 \%$ do potencial das interações foram realizadas. Os resultados mostraram, ainda, uma alta centralidade de grau das seguintes instituições: Uninove, USP, FGV (SP), UPM, UFRGS, UFSM, UFSC, UFPR, FURB e FECAP. Esse resultado apoia-se na Figura 11 que será explicada adiante, isto é, tais IES além de serem as que mais publicam no periódico, são também as mais centrais nos artigos explorados, especificamente as nove primeiras.

Pela Figura 8 visualizam-se os métodos e/ou ferramentas (mecanismos) mais utilizados pelos autores nas pesquisas relatadas nos artigos levantados da RAI no período de 2006 a 2012. 


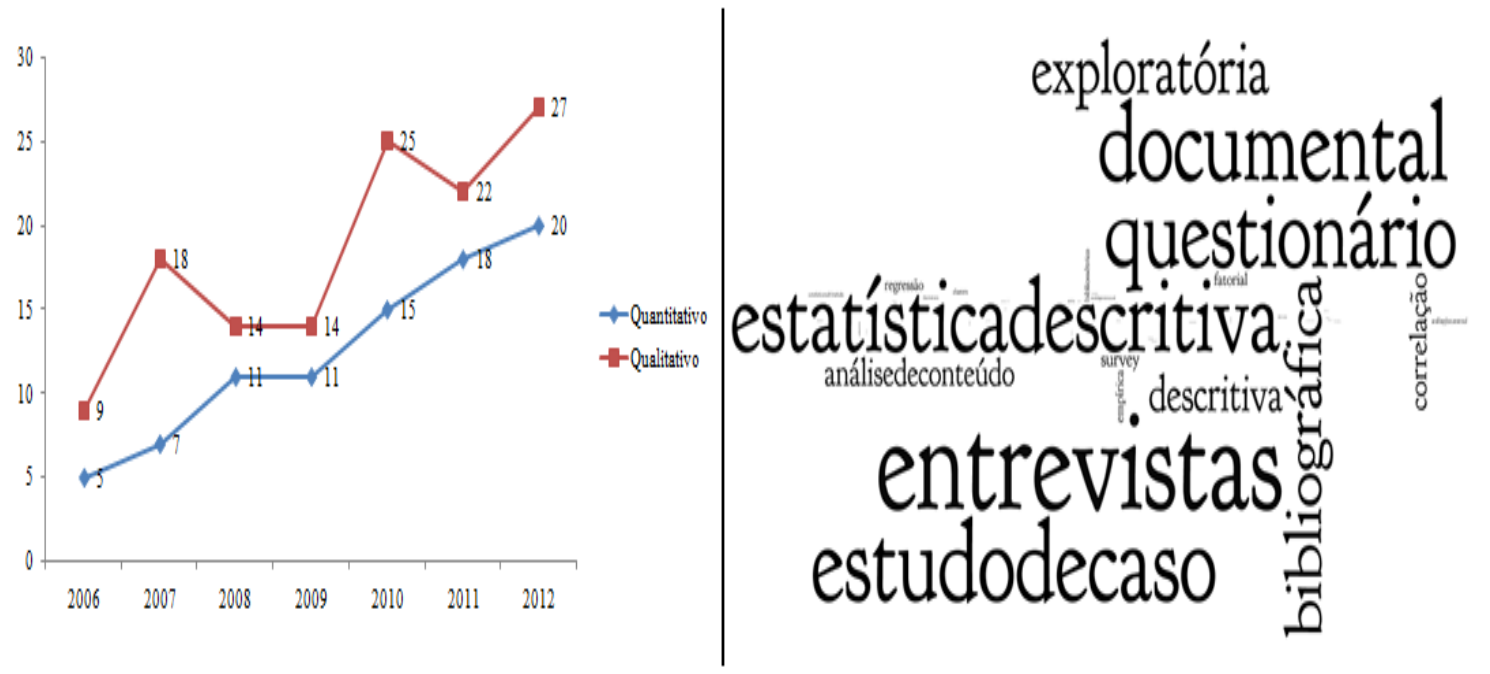

Figura 8. Frequência dos métodos de pesquisa Fonte: Dados da pesquisa

Observa-se pela Figura 8 que o método de pesquisa estudo de caso e bibliográfica se destacam nas publicações, corroborando a predominância da abordagem qualitativa nos 216 artigos publicados na RAI. Quanto aos métodos de coleta de dados, destacam-se: entrevistas, pesquisa documental e questionário. Em relação à forma de análise dos resultados, no que tange à pesquisa qualitativa, verifica-se o predomínio da técnica de análise de conteúdo e, quanto à abordagem quantitativa, destaca-se a estatística descritiva e análise de correlação.

A Figura 9 apresenta as palavras-chave mais citadas nos 216 artigos da Revista de Administração e Inovação entre os anos de 2006 e 2012.

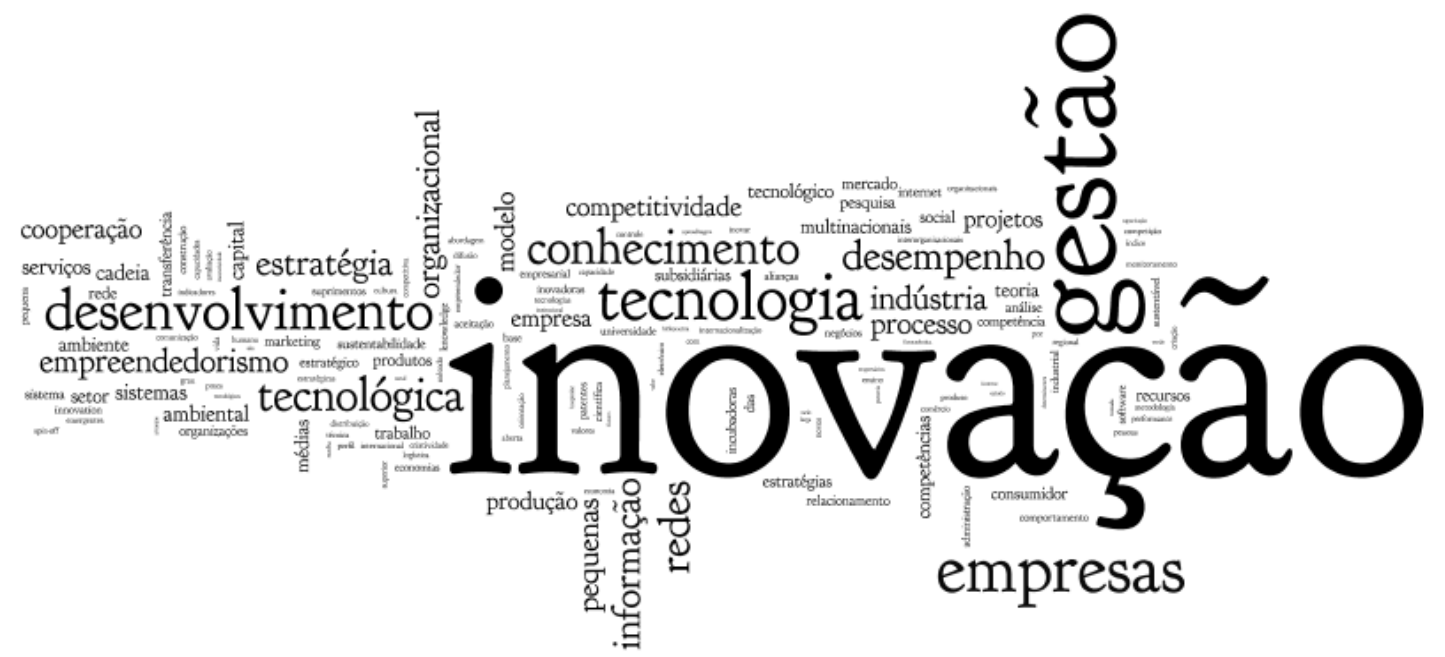

Figura 9. Frequência de palavras-chave da RAI - 2006/2012

Fonte: Dados da pesquisa 
Destacam-se as palavras-chave: inovação, gestão, tecnologia, empresas, desenvolvimento, conhecimento, dentre outras. Tais palavras refletem de alguma forma os temas tratados nos artigos investigados. Para complementar as informações visualizadas na Figura 9, a Tabela 1 a seguir apresenta os temas abordados nos 216 trabalhos investigados.

Nesse contexto, observa-se a Lei de Zipf, que calcula a frequência de ocorrências das palavras, com o intuito de criar e evidenciar o tema científico mais visualizado nos artigos científicos.

A análise dos temas é vista como fator importante para continuidade das pesquisas, o que proporciona um norte para pesquisadores experientes e, principalmente, para os iniciantes. Isso, sem dúvida, é fator determinante para o crescimento e a disseminação de futuras pesquisas. A Tabela 1 mostra a frequência dos temas nos 216 artigos explorados por ano.

É importante salientar que a classificação dos artigos por tema específico da RAI foi realizada após a leitura e a análise conjunta dos resumos de cada um dos artigos levantados nesta pesquisa (Tabela 1).

Tabela 1 - Temas mais abordados na RAI - 2006/2011

\begin{tabular}{|l|c|c|c|c|c|c|c|c|c|}
\hline Temas/Ano & $\mathbf{2 0 0 6}$ & $\mathbf{2 0 0 7}$ & $\mathbf{2 0 0 8}$ & $\mathbf{2 0 0 9}$ & $\mathbf{2 0 1 0}$ & $\mathbf{2 0 1 1}$ & $\mathbf{2 0 1 2}$ & Total & \% \\
\hline Estudos Setoriais e Tecnologia & 4 & 3 & 5 & 5 & 9 & 11 & 7 & $\mathbf{4 4}$ & $\mathbf{2 0 , 3 7}$ \\
\hline Organização e de Negócios & 3 & 5 & 3 & 4 & 4 & 3 & 2 & $\mathbf{2 4}$ & $\mathbf{1 1 , 1 1}$ \\
\hline Redes Organizacionais & 1 & 1 & 2 & 3 & 7 & 2 & 3 & $\mathbf{1 9}$ & $\mathbf{8 , 8 0}$ \\
\hline Gestão do Conhecimento & 1 & 2 & 1 & 2 & 1 & 4 & 8 & $\mathbf{1 9}$ & $\mathbf{8 , 8 0}$ \\
\hline Empreendedorismo e PMEs & & & 1 & 2 & 2 & 8 & 5 & $\mathbf{1 8}$ & $\mathbf{8 , 3 3}$ \\
\hline Ensino e Pesquisa & & 2 & & 1 & 2 & 6 & 4 & $\mathbf{1 5}$ & $\mathbf{6 , 9 4}$ \\
\hline Sustentabilidade & 1 & 3 & 1 & 3 & & 2 & 4 & $\mathbf{1 4}$ & $\mathbf{6 , 4 8}$ \\
\hline Marketing & 2 & 4 & 1 & & 4 & & 2 & $\mathbf{1 3}$ & $\mathbf{6 , 0 2}$ \\
\hline Incubação e Parques Tecnológicos & 2 & & 1 & 1 & & 3 & 6 & $\mathbf{1 3}$ & $\mathbf{6 , 0 2}$ \\
\hline Tecnologia e Competitividade & & 2 & 5 & 1 & & & 2 & $\mathbf{1 0}$ & $\mathbf{4 , 6 3}$ \\
\hline Modelos de Gestão & & 1 & 2 & 1 & 3 & & 1 & $\mathbf{8}$ & $\mathbf{3 , 7 0}$ \\
\hline $\begin{array}{l}\text { Política e Gestão de Ciência, } \\
\text { Tecnologia }\end{array}$ & & 2 & 1 & 3 & & 1 & $\mathbf{7}$ & $\mathbf{3 , 2 4}$ \\
\hline Gestão de Projetos & & 1 & 1 & 1 & 2 & & & $\mathbf{5}$ & $\mathbf{2 , 3 1}$ \\
\hline Inovação e Cultura & & & & & & 1 & 2 & $\mathbf{3}$ & $\mathbf{1 , 3 9}$ \\
\hline Economia Industrial & & 1 & & & 1 & & & $\mathbf{2}$ & $\mathbf{0 , 9 3}$ \\
\hline Transnacionalização do Capital & & & & & 2 & & & $\mathbf{2}$ & $\mathbf{0 , 9 3 \%}$ \\
\hline Total & $\mathbf{1 4}$ & $\mathbf{2 5}$ & $\mathbf{2 5}$ & $\mathbf{2 5}$ & $\mathbf{4 0}$ & $\mathbf{4 0}$ & $\mathbf{4 7}$ & $\mathbf{2 1 6}$ & $\mathbf{1 0 0 , 0 0}$ \\
\hline
\end{tabular}

Fonte: Dados da pesquisa

Nota-se que estudos setoriais e tecnologia foi o tema específico que mais se destacou nos 216 artigos analisados, seguido por inovação organizacional e de negócios; redes organizacionais; gestão 
do conhecimento; empreendedorismo e PMEs; ensino e pesquisa; sustentabilidade; marketing; incubação e parques tecnológicos; e tecnologia e competitividade. Verificou-se também o crescimento e o surgimento de novos temas nos últimos dois anos, tais como: empreendedorismo e PMEs, incubação e parques tecnológicos e gestão do conhecimento. Isso mostra o amadurecimento da Revista de Administração e Inovação com o aparecimento de outros temas, fomentando e ampliando seu foco e escopo.

As informações vão ao encontro da Lei de Bradford, na medida em que se verifica o nível de incidências das temáticas por meio de artigos publicados por revista. Tomando como referência a Revista de Administração e Inovação percebe-se que os temas são inerentes ao seu foco e escopo.

A Figura 10 mostra os autores que mais publicaram artigos durante os anos analisados.

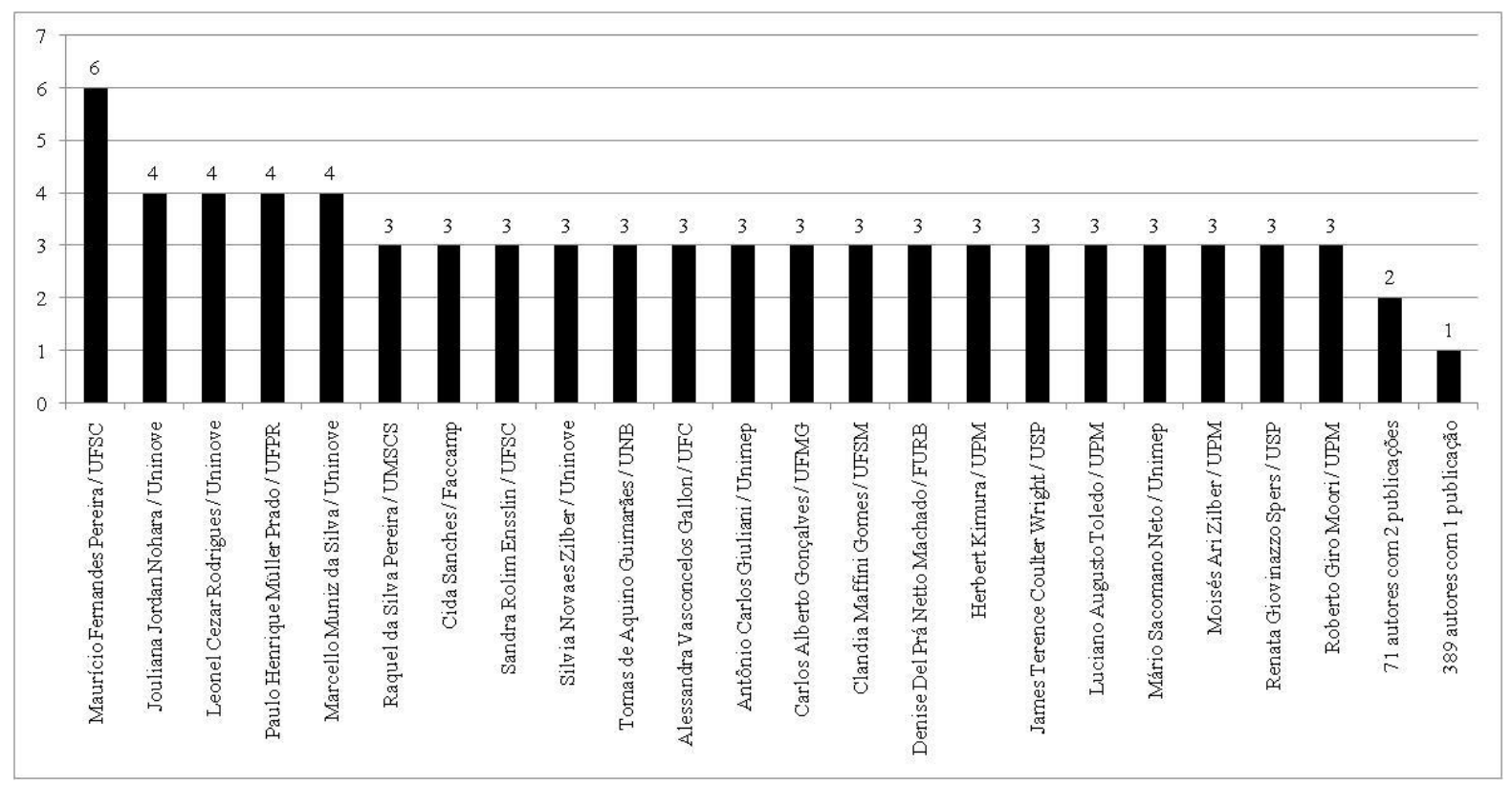

Figura 10. Autores que mais publicaram na RAI - 2006/2011

Fonte: Dados da pesquisa

Como se pode observar na Figura 10, Maurício Fernandes Pereira é o autor que mais publicou artigos na Revista de Administração e Inovação: seis artigos. Cabe mencionar ainda Jouliana Jordan Nohara, Leonel Cezar Rodrigues, Paulo Henrique Müller Prado e Marcello Muniz da Silva, autores com quatro artigos no período analisado.

Com três figuram artigos aparecem Raquel da Silva Pereira, Cida Sanches, Sandra Rolim Ensslin, Silvia Novaes Zilber, Tomas de Aquino Guimarães, Alessandra Vasconcelos Gallon, Antônio Carlos Giuliani, Carlos Alberto Gonçalves, Clandia Maffini Gomes, Denise Del Prá Netto Machado, Herbert Kimura, James Terence Coulter Wright, Luciano Augusto Toledo, Mário Sacomano Neto, Moisés Ari Zilber, Renata Giovinazzo Spers e Roberto Giro Moori. É interessante realçar que dos 22 
autores que mais publicaram, 77,27\% destes, representam as IESs mais profícuas e centrais deste estudo.

Além disso, 71 pesquisadores publicaram dois trabalhos. Mas a grande maioria, 389 autores, tiveram apenas uma publicação, o que equivale a valores percentuais de $80,71 \%$ do total de 482 que publicaram artigos na RAI ao longo dos sete anos. Esses resultados permitem compreender a Lei de Lotka, poucos pesquisadores publicam muito, enquanto que muitos publicam pouco.

A Tabela 2 resume as áreas de conhecimento estudadas pelos cinco primeiros autores que mais se destacam pela quantidade de publicações na Figura 5, evidenciando as suas respectivas áreas de atuação.

Tabela 2 - Autores que mais publicaram artigos na RAI e suas respectivas áreas temáticas 2006/2012

\begin{tabular}{|l|l|}
\hline \multicolumn{1}{|c|}{ Autores mais prolíficos } & \multicolumn{1}{|c|}{ Áreas de atuação } \\
\hline Maurício Fernandes Pereira & $\begin{array}{l}\text { Administração, Organizações e Estratégia, Comportamento } \\
\text { Organizacional, Mudança e Adaptação Estratégica, Planejamento } \\
\text { Estratégico Pessoal }\end{array}$ \\
\hline Jouliana Jordan Nohara & $\begin{array}{l}\text { Estratégia Empresarial, Administração de Recursos Humanos, Flutuações } \\
\text { Cíclicas e Projeções Econômicas, Instituições Monetárias e Financeiras do } \\
\text { Brasil }\end{array}$ \\
\hline Leonel Cezar Rodrigues & $\begin{array}{l}\text { Inovação e Estratégia em Organizações, Inteligência Competitiva, } \\
\text { Administração de Tecnologia da Informação, Administração Universitária, } \\
\text { Empreendedorismo }\end{array}$ \\
\hline $\begin{array}{l}\text { Paulo Henrique Müller } \\
\text { Prado }\end{array}$ & $\begin{array}{l}\text { Comportamento do Consumidor, Marketing de Relacionamento, } \\
\text { Marketing de Serviços, Pesquisa de Marketing, Análise de Dados Em } \\
\text { Marketing, Modelagem Em Marketing }\end{array}$ \\
\hline Marcello Muniz da Silva & Economia, Finanças Corporativas e Elaboração e Análise de Projetos. \\
\hline
\end{tabular}
Fonte: Elaborado pelos autores

A Tabela 2, mostra as temáticas que mais aparecem dentre os autores mais prolíficos: Marketing, Administração, Estratégia, Organizações e Comportamento. De uma maneira geral, esses temas de pesquisa vão ao encontro dos temas mais abordados, apresentados anteriormente na Tabela 1.

A Figura 11 apresenta as 17 IES com maior volume de publicações na Revista de Administração e Inovação de um total de 132 IES. 


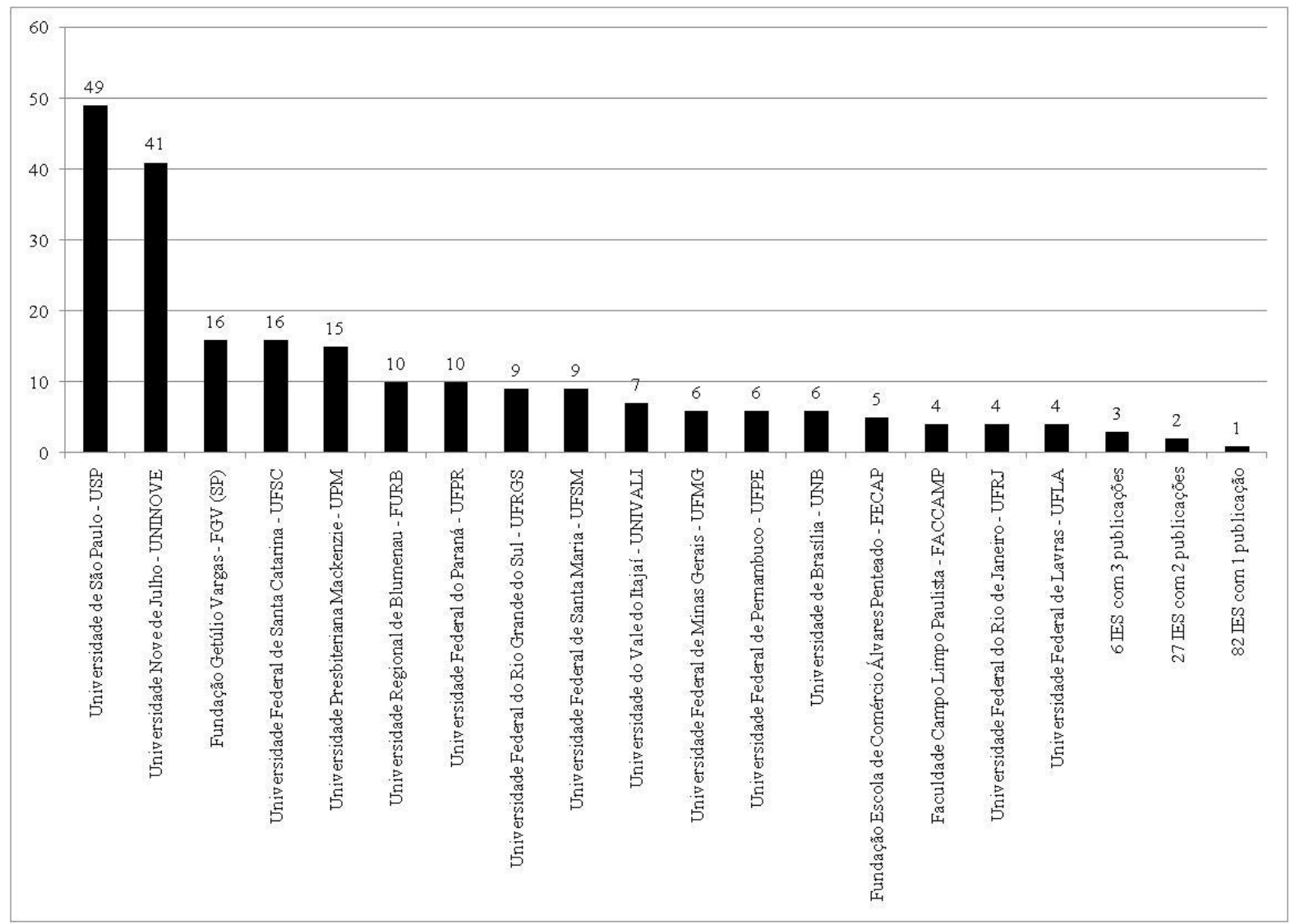

Figura 11. IES com maior publicação na RAI - 2006/2012

Fonte: Dados da pesquisa

Os resultados mostram que a Universidade de São Paulo foi a IES que mais publicou na Revista de Administração e Inovação, com 49 publicações, seguida pela Universidade Nove de Julho, com 41 artigos.

Têm também destaque: Fundação Getúlio Vargas/SP (16 artigos), Universidade Federal de Santa Catarina (16), Universidade Presbiteriana Mackenzie (15), Universidade Regional de Blumenau (10), Universidade Federal do Paraná (10), Universidade Federal do Rio Grande do Sul (9), Universidade Federal de Santa Maria (9) e Universidade do Vale do Itajaí (7). Vale destacar que, dentre as 132 IES investigadas nos 216 artigos da RAI, 23 publicaram de 3 a 49 artigos; 27 contribuíram com 2 publicações, enquanto que 82 produziram apenas um artigo, o que corresponde a cerca de $62 \%$ das instituições. 


\section{CONSIDERAÇÕES FINAIS}

Este estudo investigou a produção científica no acervo completo da Revista de Administração e Inovação no período de 2006 a 2012. Para tanto, foi realizada uma análise bibliométrica e sociométrica em 216 artigos já publicados no periódico ao longo desse período, tendo como objetivo analisar as seguintes características: autoria; redes de coautoria; referências mais citadas; rede das IES; abordagens metodológicas; frequência de palavras-chave; temas abordados; e autores e IES com maior produção.

Observou-se que $91,20 \%$ dos artigos analisados foram publicados em coautoria. Dos 216 papers investigados, aproximadamente $63 \%$ deles tem predomínio do sexo masculino. O autor Maurício Fernandes Pereira foi o mais prolífero com seis publicações, seguido de Jouliana Jordan Nohara, Leonel Cezar Rodrigues, Paulo Henrique Müller Prado e Marcello Muniz da Silva, todos com quatro artigos publicados.

Os resultados mostraram que poucos autores publicaram muito e muitos publicaram pouco, ou seja, revelam uma baixa densidade de rede, o que demonstra uma baixa integração entre os autores. Da mesma forma, observa-se uma baixa centralização quando se analisa a rede social das IES, que contempla a USP, a Uninove, a FGV (SP) e a UPM, como sendo as instituições mais centrais do estudo. No que diz respeito às citações, as obras de Porter, M. E. (72 citações), Yin, R. K. (58), Schumpeter, J. A. (46), Nelson, R. R. (40) e Pavitt, K. (39), foram as mais citadas nos 216 artigos analisados na RAI.

Em relação às abordagens metodológicas utilizadas nos artigos, constatou-se um predomínio da abordagem de pesquisa qualitativa em relação a quantitativa. Quanto à análise dos temas, a temática de estudos setoriais e tecnologia prevalece, seguida por inovação organização e de negócios, redes organizacionais, gestão do conhecimento e empreendedorismo e PMEs. Ou seja, são os temas que mais apareceram durante os sete anos de pesquisa. Esse resultado é reforçado por meio das palavraschave mais visualizadas nos 216 artigos, tais como: inovação, gestão, tecnologia, empresas, desenvolvimento, conhecimento.

Pode-se concluir que o acervo da Revista de Administração e Inovação, mesmo sendo um periódico novo, reflete o universo da produção acadêmica na área de Administração, sobretudo no tema da inovação.

Por fim, não se pode deixar de ressaltar que a pesquisa em questão refere-se ao período de sete anos. Sugere-se para futuros trabalhos um aprofundamento do estudo de redes sociais por meio de

Revista de Administração e Inovação, São Paulo, v. 10, n.4, p. 208-228, out./dez. 2013. 
outros indicadores, o que aperfeiçoaria os resultados desta pesquisa, ainda que não levem a conclusões e à generalização.

\section{REFERÊNCIAS}

Alvarenga, L. (1998). Bibliometria e arqueologia do saber de Michael Foucault - traços de identidade teórico-metodológica. Ciência da Informação, 27(3), 253-261.

Andrighi, F. F., Hoffmann, V. E., \& Andrade, M. A. R. (2011). Análise da produção científica no campo de estudo das redes em periódicos nacionais e internacionais. Revista de Administração $e$ Inovação, 8(1), 29-54.

Bobsin, D., Visentini, M. S., \& Rech, I. (2009). Em busca do estado da arte do UTAUT: ampliando as considerações sobre o uso da tecnologia. Revista de Administração e Inovação, 6(2), 99-118.

Borba, M. L., Hoeltgebaum, M., \& Silveira, A. (2011). A produção científica em empreendedorismo: análise do Academy of Management Meeting: 1954-2005. Revista de Administração Mackenzie, 12(2), 169-206.

Borges, P. C. R. (2002). Métodos quantitativos de apoio à bibliometria: a pesquisa operacional pode ser uma alternative? Ciência da Informação, 31(3), 5-17.

Didriksson, A. (2003). La sociedad del conocimiento desde la perspectiva latinoamericana. Memorias Del Encuentro de Estudios Prospectivos Región Andina: Sociedad, Educación y Desarrollo. Medellín, Colômbia, 4.

Durisin, B., Calabretta, G., \& Parmeggiani, V. (2010). The intellectual structure of product innovation research: a bibliometric study of the Journal of Product Innovation Management, 1984-2004. Journal of Product Innovation Management, 27, 437-451.

Farber, S. G., Hoeltgebaum, M., \& Klemz, B. (2011). Rede de cooperação em produção científica do Egepe de estudos sobre empreendedorismo e gestão de pequenas empresas. Revista de Administração e Inovação, 8(3), 141-161.

Fernandez-Alles, M., \& Rodríguez-Ramos, A. (2009). Intellectual structure of human resources management research: a bibliometric analysis of the journal human resource management, 1985-2005. Journal of the American Society for Information Science and Technology, 60(1), 161-175.

Ferreira, M. P. (2011). A bibliometric study on ghoshal's managing across borders. The Multinational Business Review, 19(4), 357-375.

Francisco, E. R. (2011). RAE-eletrônica: exploração do acervo à luz da bibliometria, geoanálise e redes sociais. Revista de Administração de Empresas, 51(3), 280-306. 
Freeman, L. C. (1992). Social networks and the structure experiment. In: Freeman, L. C., White, D. R., \& Romney, A. K. (Eds.). Research methods in social network analysis. (pp. 11-40). New Brunswick, NJ: Transaction Publishers.

Gnyawali, D., \& Madhavan, R. (2001). Cooperative networks and competitive dynamics: a sctrutural embeddedness perspective. Academy of Management Review, 26(3), 431-445.

Gomes, G., Machado Neto, D. D. P., \& Giotto, O. T. (2011). Análise do conteúdo dos artigos de inovação publicados nos anais do Altec, Simpoi e Enanpad (2003-2007). Revista de Administração e Inovação, 8(4), 27-44.

Hanneman, R. A., \& Riddle, M. (2005). Introduction to Social Network Methods. Riverside: University of California.

Hoffman, D. L., \& Holbrook, M. B. (1993). The intellectual structure of consumer research: a bibliometric study of author cocitations in the first 15 years of the journal of consumer research. Journal of Consumer Research, 19, 505-517.

Leal, M. S., Aguillera, F., \& Melo-Silva, L. L. (2011). Revista psicologia: organizações e trabalho uma década de sua produção científica em análise. Revista Psicologia: Organizações e Trabalho, 11(2), 6-20.

Mcmillan, G. S., \& Casey, D. L. (2007). Research note: identifying the invisible colleges of the british journal of industrial relations: a bibliometric and social network approach. British Journal of Industrial Relations, 45(4), 815-828.

Nassif, V. M. J., Silva, N. B., Ono, A. T., Bontempo, P. C., \& Tinoco, T. (2010). Empreendedorismo: área em evolução? Uma revisão dos estudos e artigos publicados entre 2000 e 2008. Revista de Administração e Inovação, 7(1), 175-192.

Ordóñez, M. G., Hernández, B. A., Hernández, C., \& Méndez, C. (2009). Análisis bibliométrico de la revista de economía institucional en sus primeros diez años. Revista de Economía Institucional, 11(20), 309-353.

Ortiz, L. C. (2002). Ferramentas alternativas para monitoramento e mapeamento automatizado do conhecimento. Ciência da Informação, 31(3), 66-76.

Parreiras, F. S., Silva, A. B. O., Matheus, R. F., \& Brandão, W. C. (2006). RedeCI: colaboração e produção científica em ciência da informação no Brasil. Perspectivas em Ciência da Informação, 11(3), 302-317.

Peleias, I. R., Wahlmann, G. C., Parisi, C., \& Antunes, M. T. P. (2010). Dez anos de pesquisa científica em controladoria no Brasil (1997-2006). Revista de Administração e Inovação, 7(1), 193217.

Pinto, D. S. P., Santos, R. N. M., \& Santos, E. M. B. (2009). Análise de citação da revista eletrônica arquivística.net: uma aplicação das técnicas bibliométricas. Em Questão, 15(1), 27-42.

Revista de Administração e Inovação (2012). Foco e escopo. Recuperado em 21 julho, 2012, de http://www.revistarai.org/ojs-2.2.4/index.php/rai/about/editorialPolicies\#focusAndScope

Revista de Administração e Inovação, São Paulo, v. 10, n.4, p. 208-228, out./dez. 2013. 
Ramos-Rodríguez, A. R., \& Ruíz-Navarro, J. (2004). Changes in the intellectual structure of strategic management research: a bibliometric study of the strategic management journal, 1980-2000. Strategic Management Journal, 25, 981-1004.

Rowley, T., Behrens, D., \& Krackhardt, D. (2000). Redundant governance structures: an analysis of structural and relational embeddedness in the stell and semiconductor industries. Strategic Management Journal, 21, 369-386.

Scott, J. (2000). Social network analysis: a handbook (2a ed.). London: Sage Publications.

Sebastián, J. (1999). Analisis de las redes de investigacion de America Latina con la Unión Europea. Revista de Ciência e Tecnologia, 3(2), 308-321.

Tomaél, M. I., \& Marteleto, R. M. (2006). Redes sociais: posições dos atores no fluxo da informação. Revista Eletrônica de Biblioteconomia e Ciência da Informação, Ed. Especial, 75-91.

Vanti, N. A. P. (2002). Da bibliometria à webometria: uma exploração conceitual dos mecanismos utilizados para medir o registro da informação e a difusão do conhecimento. Ciência da Informação, 31(2), 152-162.

Ullah, M., Butt, I. F., \& Haroon, M. (2008). The journal of ayub medical college: a 10-year bibliometric study. Health Information and Libraries Journal, 25, 116-124.

Wasserman, S., \& Faust, K. (1994). Social network analysis: methods and applications. Cambridge: Cambridge University Press.

Vitorino Filho, V. A., Sacomano Neto, M., Silva, E. M., \& Guiliani, A. C. (2012). A Produção Científica Brasileira Em Cooperação Empresarial. Revista de Administração e Inovação, São Paulo, 9(3), 175-194.

\title{
SCIENTIFIC ANALYSIS OF PRODUCTION JOURNAL OF MANAGEMENT AND INNOVATION
}

\begin{abstract}
This article investigated the scientific production of the Journal of Management and Innovation, through bibliometric and sociometric research, using descriptive statistics held in the collection of the journal. We analyzed 197 articles from 2004 to 2011. The main conclusions of this work were: Souza, M. T. S, and Pereira, R S, have high degree centrality and brokerage. Institutions of Higher Education, Uninove, USP, FGV (SP) and UFRGS have high degree centrality. In short, in general, there was poor centralization of the network of social institutions and the network of co-authorship. The research of Porter, Yin, Hamel, Nelson and Schumpeter were the most cited publications and topics that most appeared during the eight years studied were: sectoral studies and technology, business and organizational innovation, organizational networks, marketing, and entrepreneurship SMEs.
\end{abstract}

Keywords: Innovation; Administration; Bibliometrics; Sociometry.

Revista de Administração e Inovação, São Paulo, v. 10, n.4, p. 208-228, out./dez. 2013. 
Data do recebimento do artigo: 16/11/2012

Data do aceite de publicação: 23/08/2013 